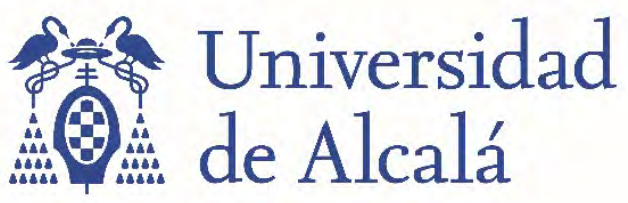

BIBLIOTECA

Document downloaded from the institutional repository of the University of Alcala: http://dspace.uah.es/

This is a postprint version of the following published document:

SENDRA, R., SEVILLA, D. and VILLARINO, C., 2015. Some Results on the Surjectivity of Surface Parametrizations. Computer Algebra and Polynomials: Applications of Algebra and Number Theory, 8942, pp. 192203.

Available at http://dx.doi.org/10.1007/978-3-319-15081-9 11

(C) 2015 Springer International Publishing Switzerland
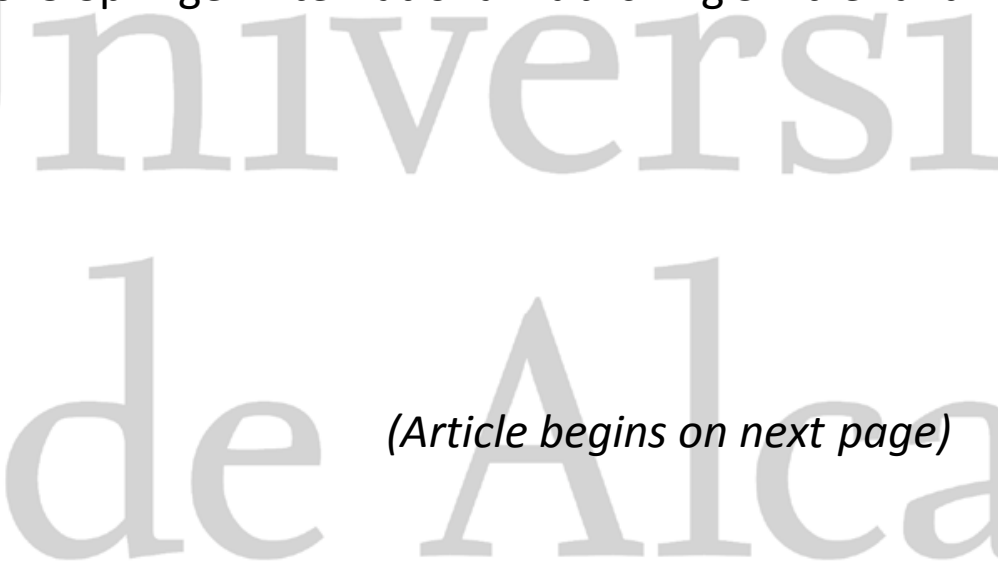

(Article begins on next page)

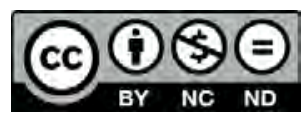

This work is licensed under a

Creative Commons Attribution-NonCommercial-NoDerivatives

4.0 International License. 
The final journal version of this paper appears in:

\title{
Some results on the surjectivity of surface parametrizations
}

\author{
J. Rafael Sendra ${ }^{1}$, David Sevilla ${ }^{2}$, and Carlos Villarino ${ }^{1}$ \\ 1 Dept. of Physics and Mathematics \\ University of Alcalá \\ Ap. Correos 20, E-28871 \\ Alcalá de Henares (Madrid), Spain \\ rafael.sendra@uah.es, carlos.villarino@uah.es \\ http://www2.uah.es/rsendra \\ 2 University Center of Mérida \\ Av. Santa Teresa de Jornet 38 \\ E-06800 Mérida (Badajoz), Spain \\ sevillad@uex.es \\ http://www.davidsevilla.com
}

\begin{abstract}
This paper deals with the decision problem of the surjectivity of a rational surface parametrization. We give sufficient conditions for a parametrization to be surjective, and we describe different families of parametrizations that satisfy these criteria. In addition, we consider the problem of computing a superset of the points not covered by the parametrization. In this context, we report on the case of parametrizations without projective base points and we analyze the particular case of rational ruled surfaces.
\end{abstract}

Keywords: Rational algebraic surface, normality, ruled surfaces, base points

\section{Introduction}

Often, when motivating the applicability of rational surfaces, one claims that providing a different representation to the implicit one (for example a rational parametrization representation) is very useful in many particular applied problems, for instance in computer aided geometric desing (see [9] and [8]). Examples of this are plotting images in computer screens, curve or surface integration, intersection of varieties, etc. Nevertheless, even though this is true, one must add that, for the parametrization to be feasibly useful, it must satisfy certain properties. For instance, if we are using a parametrization for plotting, and the parametrization is given over the complex numbers, how do we know whether the variety is indeed real? What parameter values must be put into the parametrization in order to get real points? Of course, this is not a problem if the input parametrization is given with real coefficients; this problem has been studied in [1], [2], and [12]. 
A similar phenomenon can arise when computing the intersection of two surfaces where one of them is given parametrically: how do we know that the parametrization is not missing essential information for the problem we are dealing with? This is, indeed, the central topic of this paper: surjectivity (also called normality) of rational surface parametrizations. Let us illustrate by an example the situation described above.

Example 1. We consider the surfaces $S_{1}$ and $S_{2}$ defined by

$$
F_{1}(x, y, z)=x^{2} y^{2} z-2 x y z-x^{2}+z
$$

and

$$
F_{2}(x, y, z)=-x^{3} y^{2} z+x^{2} y^{2} z+2 x^{2} y z+x^{3}-2 x y z-x^{2}+z
$$

respectively. If we compute a Gröbner basis of $\left(F_{1}, F_{2}\right)$ we get $\left\{z^{2}, x z, x^{2}-z\right\}$, and so $S_{1} \cap S_{2}$ consists of the line $z=x=0$. However, if we take the rational parametrization

$$
\mathcal{P}(s, t)=\left(\frac{1}{t}, s+t, \frac{1}{s^{2}}\right)
$$

of $S_{1}$ and we substitute it in $F_{2}$ we get $1 /\left(t s^{2}\right)$ and hence no intersection point. Nevertheless, if we observe that $F_{1}$ is linear in $z$ we can also consider the parametrization

$$
\mathcal{Q}(s, t)=\left(s, t, \frac{s^{2}}{s^{2} t^{2}-2 s t+1}\right)
$$

of $S_{1}$. Substituting this new parametrization in $S_{2}$ we get

$$
\frac{s^{3}}{s^{2} t^{2}-2 s t+1} \text {. }
$$

So the intersection of $S_{1}$ and $S_{2}$ is $\mathcal{Q}(0, t)=(0, t, 0)$, the expected line. The difference between $\mathcal{P}(s, t)$ and $\mathcal{Q}(s, t)$ is that the second one is surjective. See Remark 3 in relation to the parametrization $\mathcal{Q}$.

The problem of deciding the normality of a surface parametrization (i.e. whether it is surjective over the algebraic surface) can be attacked by means of elimination theory techniques, but a direct application of these can be too complex in terms of time. Moreover, deciding whether a given rational surface admits a normal parametrization is, at least to our knowledge, an open problem. In this article we approach the problem by, on one hand, giving sufficient conditions for the normality of an input rational surface parametrization; and, on the other, for general rational surfaces and under certain conditions, as well as for ruled surfaces, we describe explicitly a convenient superset of the complementary of the image in the surface.

In situations where we cannot assure normality, an alternative approach, proposed in [15], is to cover the algebraic surface with a finite number of affine parametrizations. In projective space this is possible by [4]. 
When dealing with coverings we need to assume that the parametrization has no projective base points. Only in some cases, like ruled surfaces, there has been progress in base point removal, see [6] and its reference [14]; see also [3] for the surface implicitization problem. Therefore in most situations it is assumed that the given parametrization has none base points, as we do here. As an intermediate step, one can reparametrize in such a way that all affine base points are sent to infinity, see [16] for details.

Ruled surfaces are of particular relevance in applications. We show how, given any rational parametrization of a ruled surface, one can reparametrize it so that provided the new parametrization does not have affine points, then it is normal or the set of missing points is contained in a line that can be made explicit.

Our coefficient field is algebraically closed of characteristic zero; for other fields (for example $\mathbb{R}$, of obvious interest) the curve case already suffers from complications that make the analysis very difficult, see [5], [13].

The structure of the article is as follows: in Section 2 we show how to calculate algebraic supersets of the complement of a given parametrization. In Section 3 we present criteria for normality. Section 4 deals with the ruled case.

\section{Computation of critical sets}

Let us fix some notation through a few definitions. In the sequel, we use the following terminology: a parametrization takes its values in two-dimensional space in the variables $s, t$ into three-dimensional space in the variables $x, y, z$.

Definition 1. Let $\mathbb{K}$ be an algebraically closed field of characteristic zero and $S \subset \mathbb{K}^{3}$ an affine algebraic surface. A parametrization of $S$ is a triple of rational functions that determines a rational dominant map

$$
\begin{aligned}
\mathcal{P}: & \mathbb{K}^{2}-\rightarrow \\
(s, t) & \mapsto\left(\frac{p_{1}(s, t)}{q(s, t)}, \frac{p_{2}(s, t)}{q(s, t)}, \frac{p_{3}(s, t)}{q(s, t)}\right) .
\end{aligned}
$$

We assume w.l.o.g. that $\operatorname{gcd}\left(p_{1}, p_{2}, p_{3}, q\right)=1$. We denote as $\bar{S}$ the projective closure of $S$ in $\mathbb{P}^{3}(\mathbb{K})$. The function $\mathcal{P}$ has a projective counterpart, $\overline{\mathcal{P}}$ :

$$
\begin{aligned}
\overline{\mathcal{P}}: & \mathbb{P}^{2}(\mathbb{K}) \quad-\rightarrow \quad \mathbb{P}^{3}(\mathbb{K}) \\
\mathbf{s}=(s: t: u) & \mapsto\left(\overline{p_{1}}(\mathbf{s}): \overline{p_{2}}(\mathbf{s}): \overline{p_{3}}(\mathbf{s}): \bar{q}(\mathbf{s})\right)
\end{aligned}
$$

where the four components are the polynomial homogenizations of the numerators and denominator of $\mathcal{P}$ such that their gcd is 1 and they have the same degree. Note that $\overline{\mathcal{P}}$ may be undefined at some points of $\mathbb{P}^{2}(\mathbb{K})$, since its four components may have a common zero.

Definition 2. The common zeros of the components of $\overline{\mathcal{P}}$ are called projective base points. Such a point $(s: t: u)$ is also called an affine base point if $u \neq 0$. 
Since the gcd of the four homogeneous polynomials is 1, by Bézout's theorem it follows that there can be at most finitely many projective base points.

Definition 3. An (affine) surface parametrization is called normal if it is surjective on $S$, that is, for every $p \in S$ there exist $s_{0}, t_{0} \in \mathbb{K}$ such that $\mathcal{P}\left(s_{0}, t_{0}\right)=p$.

Definition 4. Let $\mathcal{P}$ be a parametrization that is not normal. A closed proper subset $C \subset S$ is called a critical set of $\mathcal{P}$ if $C \supset S \backslash \mathcal{P}\left(\mathbb{K}^{2}\right)$.

Example 2. In Example 1, the line $z=x=0$ is a critical set for $\mathcal{P}$. Any (reducible) curve on the surface that contains that line is also a critical set.

Example 3. The cone $x^{2}+y^{2}=z^{2}$ has the parametrization

$$
\mathcal{P}(s, t)=\left(\frac{2 s t}{1+t^{2}}, \frac{s\left(1-t^{2}\right)}{1+t^{2}}, s\right)
$$

that has the critical set $x=y+z=0$. The complement of the image is that line minus the origin, since $\mathcal{P}(0, t)=(0,0,0)$ for any $t \neq \pm \sqrt{-1}$.

Now we give explicit descriptions of a critical set. See [15] for details. It is important to remark that the set of missing points may be finite, but we do currently know how to detect that case or compute a finite critical set when it is possible to do so, although eventually the method may generate zero-dimensional outputs (see Examples 5 and 6).

Theorem 1. Let $\mathcal{P}$ be a non-normal parametrization of a surface $S$ without projective base points. In the notation of Definition 1 , let $n=\operatorname{deg}(q)$ and $l_{i}=$ $\operatorname{deg} q-\operatorname{deg} p_{i}$ for $i=1,2,3$. Necessarily $l_{i} \geq 0$ by the hypothesis. Let $P_{i, n-l_{i}}(s, t)$ be the homogeneous form of highest degree in $p_{i}(s, t)$ and $Q_{n}(s, t)$ that of $q(s, t)$.

Let $\delta_{i, j}$ denote the Kronecker delta. Define

$$
C_{1}(s)=\left(\frac{P_{1, n-l_{1}}(s, 1)}{Q_{n}(s, 1)} \cdot \delta_{l_{1}, 0}, \frac{P_{2, n-l_{2}}(s, 1)}{Q_{n}(s, 1)} \cdot \delta_{l_{2}, 0}, \frac{P_{3, n-l_{3}}(s, 1)}{Q_{n}(s, 1)} \cdot \delta_{l_{3}, 0}\right),
$$

and

$$
p=\left(\frac{P_{1, n-l_{1}}(1,0)}{Q_{n}(1,0)} \cdot \delta_{l_{1}, 0}, \frac{P_{2, n-l_{2}}(1,0)}{Q_{n}(1,0)} \cdot \delta_{l_{2}, 0}, \frac{P_{3, n-l_{3}}(1,0)}{Q_{n}(1,0)} \cdot \delta_{l_{3}, 0}\right) .
$$

Then $S=\mathcal{P}\left(\mathbb{K}^{2}\right) \cup C_{1}(\mathbb{K}) \cup\{p\}$. In particular, the rational curve

$$
C_{1}(\mathbb{K}) \cup\{p\}=\{\overline{\mathcal{P}}(s: t: 0) \mid(s: t) \in \mathbb{P}(\mathbb{K})\} \cap S .
$$

is a critical set.

Remark 1. It is worth noting that by a Gröebner basis computation we can decide if a given point in space belongs to the image of the parametrization. The same technique works if we want to test the points of a curve on the surface, whether parametrically or implicitly given. 
Remark 2. In the particular case when $\mathcal{P}$ is proper (i.e. injective) with inverse $\mathcal{Q}$, the points not in the image are contained in the curves defined by the denominators of $\mathcal{P}$ and the denominators of $\mathcal{P}(\mathcal{Q})$. More precisely, one can proceed as follows:

1. Compute a representant of the inverse of $\mathcal{P}$; say

$$
\mathcal{Q}(x, y, z)=\left(\frac{A_{1}(x, y, z)}{B_{1}(x, y, z)}, \frac{A_{2}(x, y, z)}{B_{2}(x, y, z)}\right) .
$$

2. Compute the denominators $D_{i}(x, y, z)$ of $\mathcal{P}(\mathcal{Q}(x, y, z))$.

3. The intersection of the algebraic surface and the algebraic set $V\left(\operatorname{lcm}\left(D_{1}, D_{2}, D_{3}, B_{1}, B_{2}\right)\right)$ is a lower-dimensional algebraic set containing the set of non-reachable points. (Here $V\left(F_{1}, \ldots, F_{s}\right)$ denotes the algebraic set defined over $\mathbb{K}$ by the polynomials $\left\{F_{1}, \ldots, F_{m}\right\} \subset \mathbb{K}[x, y, z]$.)

The following example illustrates this.

Example 4. Let us consider the revolution surface $\mathcal{S}$ given parametrically by

$$
\mathcal{P}(s, t)=\left(\frac{2 s}{s^{2}+1} \frac{t-1}{t+1}, \frac{\left(1-s^{2}\right)}{s^{2}+1} \frac{t-1}{t+1}, \frac{t+1}{t-1}\right)
$$

obtained by rotating the hyperbola $\mathcal{C}$, of implicit equation $y z=1$ given parametrically by $\left(0, \frac{t-1}{t+1}, \frac{t+1}{t-1}\right)$, around the $z$-axis. In order to analyze the missing points of the surface, we first observe that the hyperbola itself has only a missing point $P=(0,1,1)$ which corresponds to the limit when $t \rightarrow \infty$ (see [13]). Then it is logical to think that the circle that this point generates is a circle not reachable by $\mathcal{P}$; direct calculations confirm it. On the other hand, another candidate curve for missing points could be the limit curve $\left(0,-\frac{t-1}{t+1}, \frac{t+1}{t-1}\right)$ (obtained as $s \rightarrow \infty)$ and that is, in the plane $y z$, the curve symmetric of the hyperbola $\mathcal{C}$ with respect the $z$-axis. Again, direct calculations show that no point of this curve is reachable by $\mathcal{P}$. Now, in order to see that there are no more missing points we compute a critical set by means of the inverse of $\mathcal{P}$. From a Gröbner basis for the ideal

$$
\text { (numer } \left.\left(p_{1}-x\right), \operatorname{numer}\left(p_{2}-y\right), \operatorname{numer}\left(p_{3}-z\right), w\left(s^{2}+1\right)\left(t^{2}-1\right)-1\right)
$$

we can choose the representative of the inverse of $\mathcal{P}$

$$
\mathcal{Q}(x, y, z)=\left(\frac{z+1}{z-1}, \frac{x z}{y z+1}\right) .
$$

The curves defined by the denominators of $\mathcal{Q}$ and $\mathcal{P}(\mathcal{Q})$ over $\mathcal{S}$ are contained in

$$
z(z-1)(y z+1)\left(x^{2} z^{2}+y^{2} z^{2}+2 y z+1\right)=0, \quad x^{2} z^{2}+y^{2} z^{2}-1=0 .
$$

The prime decomposition of this variety is $(x, y z+1) \cup\left(z-1, x^{2}+y^{2}-1\right)$. Then, a critical set it is formed by the hyperbola $x=y z+1=0$ and the circle $z-1=x^{2}+y^{2}-1=0$; among the choices of the inverse, this is the best possible outcome. 
Example 5. We consider the parametrization

$$
\mathcal{P}(s, t)=\left(\frac{t^{2}}{s^{2}+t^{2}}, \frac{s}{s^{2}+t^{2}}, \frac{s t}{s^{2}+t^{2}}\right)
$$

of the cylinder $x^{2}+z^{2}-x=0$. A representative of the inverse of $\mathcal{P}$ is

$$
\left(\frac{1-x}{y}, \frac{z}{y}\right) \text {. }
$$

Applying the procedure described in Remark 2, the critical set obtained is the point $(1,0,0)$. This point is reachable as $\mathcal{P}(0,1)$, thus the parametrization is in fact normal.

Example 6. We consider the parametrization

$$
\mathcal{P}(s, t)=\left(\frac{t^{2}}{s^{3}+t^{3}}, \frac{s}{s^{3}+t^{3}}, \frac{t}{s^{3}+t^{3}}\right)
$$

of the quintic surface of equation $x^{2} y^{3}+x^{2} z^{3}-z^{4}=0$. A representative of the inverse of $\mathcal{P}$ is

$$
\left(\frac{x y}{z^{2}}, \frac{x}{z}\right) \text {. }
$$

From this the critical set obtained is the point $(0,0,0)$. From the parametrization, it is clear that the origin is not reachable. Thus the critical set is optimal.

In the following example the critical set given by Theorem 1 is smaller that the one generated by Remark 2 .

Example \%. We consider the parametrization

$$
\mathcal{P}=\left(\frac{t^{2}}{s^{2}+1}, \frac{s^{2}+t}{s^{2}+1}, \frac{t^{2}+s}{s^{2}+1}\right) .
$$

Observe that it has no projective base points. The surface parametrized by $\mathcal{P}(s, t)$ is defined by the degree 4 polynomial

$$
\begin{aligned}
& x^{4}-4 x^{3} y-6 x^{3} z+2 x^{2} y^{2}+8 x^{2} y z+7 x^{2} z^{2}-4 x y^{2} z-4 x y z^{2}- \\
& -4 x z^{3}+y^{4}+2 y^{2} z^{2}+z^{4}+3 x^{3}-2 x^{2} y-6 x^{2} z-x y^{2}+4 x y z+ \\
& +3 x z^{2}-2 y^{3}-2 y z^{2}+2 x y+y^{2}-x .
\end{aligned}
$$

The inverse of $\mathcal{P}$ can be expressed as

$$
\left\{s=\frac{x^{2}-2 x y-x z+2 y z+x-z}{x^{2}-2 x z-y^{2}+z^{2}+2 y-1}, t=\frac{x^{2}-x y-2 x z+y^{2}+z^{2}+x-y}{x^{2}-2 x z-y^{2}+z^{2}+2 y-1}\right\} .
$$

Thus Remark 2 provides, as a critical set, the variety

$$
V(-2 y+x+1,3 x-1-2 z) \cup V(y-1, x-z) \cup V(-2 y+x+1, x+1-2 z) .
$$

However, Theorem 1 reduces the critical set to $V(y-1, x-z)$. 


\section{Criteria for normality}

We will describe sufficient conditions on a rational surface parametrization to be normal. Note that the composition of a normal parametrization with an affine transformation of $\mathbb{K}^{3}$ is again normal. So the results below are also valid applying affine transformations to the parmatrizations.

Taking into account the inverse-based method for computing critical sets described in Remark 2, we have the following criterium.

Proposition 1. Every proper polynomial parametrization with polynomial inverse is normal.

Example 8. We consider the surface $S$ defined by $x^{4}-2 x^{2} y+y^{2}+x-z$. It can be parametrized by $\mathcal{P}(s, t)=\left(t, t^{2}+s, s^{2}+t\right)$. This parametrization is proper and its inverse can be expressed polynomially as $\left(y-x^{2}, x\right)$. Therefore, $\mathcal{P}(s, t)$ is normal.

Corollary 1. A parametrization of the form

$$
\mathcal{P}(s, t)=(a s+b, c t+A(s), B(s, t))
$$

where $a c \neq 0, A \in \mathbb{K}[s]$ and $B \in \mathbb{K}[s, t]$ is normal.

Proof. Note that $\mathcal{P}(s, t)$ is polynomial and proper, and its inverse can be expressed as

$$
\left(\frac{x-b}{a}, \frac{y-A\left(\frac{x-b}{a}\right)}{c}\right)
$$

which is polynomial.

More generally, one has the next corollary.

Corollary 2. A parametrization of the form

$$
\mathcal{P}(s, t)=\left(\varphi_{1}(s, t), \varphi_{2}(s, t), B(s, t)\right),
$$

where $\left(\varphi_{1}, \varphi_{2}\right)$ is an automorphism of $\mathbb{K}^{2}$, and $B \in \mathbb{K}[s, t]$, is normal.

Proof. The inverse of $\mathcal{P}(s, t)$ can be expressed as

$$
\left(\varphi_{1}(s, t), \varphi_{2}(s, t)\right)^{-1}
$$

which is polynomial.

Remark 3. Every irreducible surface $\mathcal{S}$ defined by a polynomial $F(x, y, z)$ with degree 1 with respect to one of the variables is rational. Assuming w.l.o.g. that $\operatorname{deg}_{z}(F)=1$, then $F$ can be expressed as $F=p(x, y)-z q(x, y)$ where $\operatorname{gcd}(p, q)=$ 1 , and $\mathcal{S}$ can be easily parametrized as

$$
\mathcal{P}(s, t)=\left(s, t, \frac{p(s, t)}{q(s, t)}\right) .
$$


The analysis of the normality of this parametrization is also very simple: $\mathcal{P}$ is a normal parametrization of $\mathcal{S}$ if and only if $V(p(s, t), q(s, t))$ is empty. Indeed, if $V(p(s, t), q(s, t))=\bigcup\left\{\left(s_{i}, t_{i}\right)\right\}$, then the lines $\left(s_{i}, t_{i}, \lambda\right)_{\lambda \in \mathbb{K}}$ are in $\mathcal{S}$ and are not reachable. On the other hand, if $\mathcal{P}$ is not normal there exists a point $\left(x_{0}, y_{0}, z_{0}\right) \in$ $\mathcal{S}$ not reachable by $\mathcal{P}$; this implies that $q\left(x_{0}, y_{0}\right)=p\left(x_{0}, y_{0}\right)=0$.

In particular, the parametrization $\mathcal{Q}$ in Example 1 is normal because $V\left(s, s^{2} t^{2}-\right.$ $2 s t-1)=\emptyset$.

The following criterium can be found in Theorem 5 in [15]. It applies to situations where there is an absence of projective base points.

Proposition 2. Let $\mathcal{P}(s, t)$ have no projective base points. If

$$
\max \left\{\operatorname{deg}\left(p_{1}\right), \operatorname{deg}\left(p_{2}\right), \operatorname{deg}\left(p_{3}\right)\right\}>\operatorname{deg}(q)
$$

then $\mathcal{P}(s, t)$ is normal.

Example 9. By Proposition 2, we know that the parametrization

$$
\mathcal{P}(s, t)=\left(\frac{s^{2}}{s+2 t}, \frac{t^{2}}{s-2 t}, \frac{s t+1}{s+2 t}\right)
$$

is normal.

Corollary 3. A parametrization of the form

$$
\mathcal{P}(s, t)=\left(A_{1}(s), A_{2}(t), A_{3}(s, t)\right)
$$

where $A_{1}, A_{2}$ are non-constant polynomials of the same degree, $A_{3} \in \mathbb{K}[s, t]$ with $\operatorname{deg}\left(A_{3}\right) \leq \operatorname{deg}\left(A_{1}\right)$, is normal.

Proof. Let $A_{1}=a_{n} s^{n}+\cdots+a_{0}, A_{2}=b_{n} t^{n}+\cdots+b_{0}$. Let $\mathcal{P}^{H}(s, t, u)$ be the projectivization of $\mathcal{P}(s, t)$. Then, $\mathcal{P}^{H}(s, t, 0)=\left(a_{n} s^{n}: b_{n} t^{n}: \alpha: 0\right)$ for some $\alpha \in \mathbb{K}[s, t]$. So, the parametrization does not have projective base points. Now the result follows from Proposition 2.

Corollary 4. A parametrization without affine base points of the form

$$
\mathcal{P}(s, t)=\left(\frac{p_{1}}{q}, \frac{p_{2}}{q}, \frac{p_{3}}{q}\right)
$$

where

$$
\begin{aligned}
& p_{1}=a_{n} t^{n}+P_{1}^{*}(s, t), \\
& p_{2}=b_{n} s^{n}+P_{2}^{*}(s, t),
\end{aligned}
$$

such that $P_{1}^{*}, P_{2}^{*}$ and $q$ have degree strictly less than $n$, and $p_{3}$ has degree $\leq n$, is normal.

Proof. Once again, $\mathcal{P}^{H}(s, t, 0)=\left(a_{n} s^{n}: b_{n} t^{n}: \alpha: 0\right)$ for some $\alpha \in \mathbb{K}[s, t]$ so there are no projective base points. 
The next criterium is based on the notion of pseudo-normality, a concept introduced in [11]. Let I be the implicitization ideal of $\mathcal{P}(s, t)$, that is the ideal in $\mathbb{K}[W, s, t, x, y, z]$ generated by $\left\{q(s, t) x-p_{1}(s, t), q(s, t) y-p_{2}(s, t), q(s, t) z-\right.$ $\left.p_{3}(s, t), q W-1\right\}$. We consider the maps $\Pi_{s}: \mathbb{K}^{5} \rightarrow \mathbb{K}^{4} ;(s, t, x, y, z) \mapsto(t, x, y, z)$ and $\Pi_{t}: \mathbb{K}^{4} \rightarrow \mathbb{K}^{3} ;(t, x, y, z) \mapsto(x, y, z)$. Let $V$ be the variety in $\mathbb{K}^{5}$ defined by $\left\{q(s, t) x-p_{1}(s, t), q(s, t) y-p_{2}(s, t), q(s, t) z-p_{3}(s, t)\right\}$. In this situation, we say that $\mathcal{P}(s, t)$ is pseudo-normal if

$$
S \subset \pi_{t}\left(\pi_{s}(V)\right)
$$

The next result can be found in Corollary 4.4. in [11].

Proposition 3. A pseudo-normal polynomial parametrization is normal.

To finish this section, we present a family of normal parametrizable surfaces that do not come from the previous criteria. In Figure 1 we see one of them. As the image suggests, they correspond to surfaces having three independent planes being asymptotes of the surface.

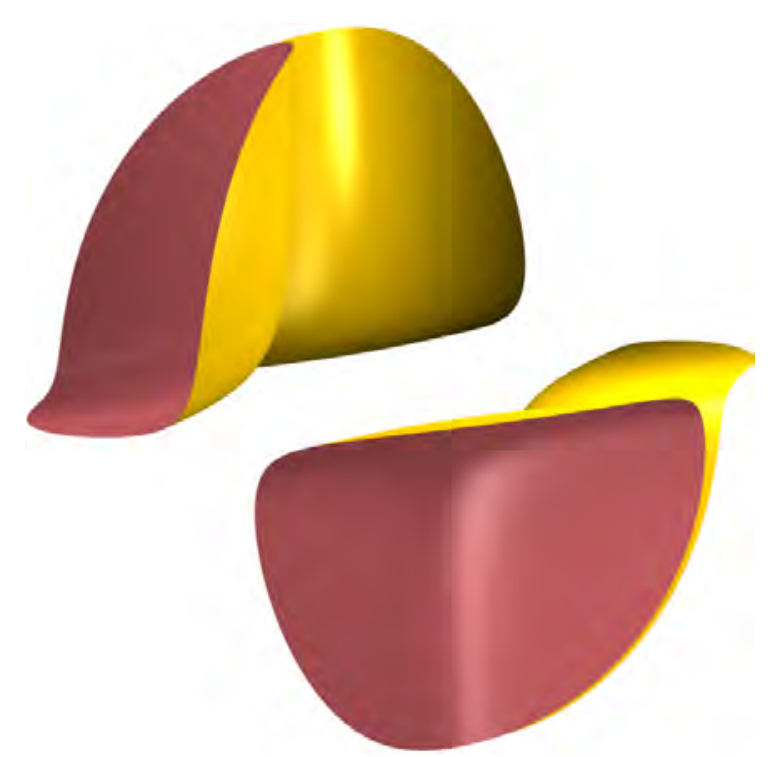

Fig. 1. The surface $x^{4} y^{5} z^{3}=1$

Proposition 4. Let $\lambda \neq 0$ and let $S$ be the surface defined by

$$
L_{1}(x, y, z)^{n} L_{2}(x, y, z)^{m} L_{3}(x, y, z)^{k}=\lambda
$$

where $L_{i}$ are three linearly independent linear forms with natural exponents. Then $S$ can be normally parametrized. 
Proof. W.l.o.g. we can assume, after a suitable affine linear change, that $S$ is given by $x^{n} y^{m} z^{k}=\lambda$. We consider the parametrization

$$
\mathcal{P}(s, t)=\left(s^{k}, t^{k}, \sqrt[k]{\lambda} /\left(s^{n} t^{m}\right)\right) .
$$

Since $\lambda \neq 0$, the affine surface does not intersect the coordinate planes $x=0$, $y=0$, and $z=0$. Now observe that, if $(a, b, c) \in S$, then $(a, b, c)=\mathcal{P}(\sqrt[k]{a}, \sqrt[k]{b})$.

\section{Critical set of ruled surfaces}

Our starting point is a parametrization of a ruled surface. In [10] methods to determine if a surface is ruled are presented, including the computation of a parametrization of the surface in the form $A(t)+s B(t)$. We will assume without loss of generality that any ruled surface is given in this form.

Lemma 1. A parametrization as above can be reparametrized into the form

$$
\mathcal{P}(s, t)=\left(\frac{r_{1}(s)+t p_{1}(s)}{q(s)}, \frac{r_{2}(s)+t p_{2}(s)}{q(s)}, \frac{r_{3}(s)+t p_{3}(s)}{q(s)}\right)
$$

where $\operatorname{gcd}\left(p_{1}, p_{2}, p_{3}\right)=1, \operatorname{gcd}\left(r_{1}, r_{2}, r_{3}, q\right)=1, \operatorname{deg}\left(r_{1}\right)=\operatorname{deg}\left(r_{2}\right)=\operatorname{deg}\left(r_{3}\right)=$ $\operatorname{deg}(q)=m$ and $\operatorname{deg}\left(p_{1}\right)=\operatorname{deg}\left(p_{2}\right)=\operatorname{deg}\left(p_{3}\right)=n$.

Proof (Sketch). It suffices to follow these steps:

- Put a common denominator.

- By a Möbius transformation, the degrees of the $r, p, q$ are made equal.

- With the change $t \leftarrow t / \operatorname{gcd}\left(p_{i}\right)$, the $p_{i}$ are made coprime.

The following theorem describes simple critical sets of ruled surface parametrizations given as in Lemma 1, and under the assumption of not having affine base points.

Theorem 2. Let $\mathcal{P}$ be a ruled surface parametrization in the form provided by Lemma 1 without affine base points. Then the following line is a critical set of $\mathcal{P}:$

$$
\left\{\begin{array}{l}
p_{2 n}\left(x q_{m}-r_{1 m}\right)-p_{1 n}\left(y q_{m}-r_{2 m}\right)=0 \\
p_{3 n}\left(x q_{m}-r_{1 m}\right)-p_{1 n}\left(z q_{m}-r_{3 m}\right)=0
\end{array}\right.
$$

where $p_{i n}, r_{i m}$ and $q_{m}$ are the leading coefficients of the polynomials $p_{i}, r_{i}$ and $q$ respectively. Moreover, a parametrization of this line is

$$
\left(\frac{r_{1 m}+\lambda p_{1 n}}{q_{m}}, \frac{r_{2 m}+\lambda p_{2 n}}{q_{m}}, \frac{r_{3 m}+\lambda p_{3 n}}{q_{m}}\right), \quad \lambda \in \mathbb{K}
$$

that can be obtained from $\mathcal{P}$ considering $\mathcal{Q}(0, \lambda)$ in $\mathcal{Q}(s, t)=\mathcal{P}\left(\frac{1}{s}, \frac{t}{s^{m-n}}\right)$. 
Proof (Sketch). In the ring $\mathbb{K}[x, y, z, s, t, w]$ we consider the ideal

$$
I=\left(r_{1}(s)+t \cdot p_{1}(s)-x \cdot q(s), \ldots, \ldots, w \cdot q(s)-1\right) .
$$

Then Image $(\mathcal{P})=\pi(V(I))$ where $\pi: \mathbb{K}^{6} \rightarrow \mathbb{K}^{3}: \pi(x, y, z, s, t, w)=(x, y, z)$. Repeated application of the Extension Theorem (see in [7], also Exercise 6.3.7 in p. 283) proves the result.

Remark 4. The concept of pseudonormality introduced in [11] corresponds to the case where it is possible to extend a surface point to $s, t$ but possibly not to $w$.

In the next example Theorem 2 provides a better critical set than Remark 2 .

Example 10. We consider the parametrization

$$
\mathcal{P}(s, t)=\left(\frac{s+t\left(s^{2}+1\right)}{s+1}, \frac{s+3+t\left(s^{2}-2\right)}{s+1}, \frac{s+2+t\left(s^{2}-3\right)}{s+1}\right)
$$

that parametrizes the cubic surface defined by the polynomial

$$
\begin{aligned}
& x^{3}-8 x^{2} y+7 x^{2} z+16 x y^{2}-32 x y z+15 x z^{2}+16 y^{2} z-24 y z^{2}+ \\
& +9 z^{3}-6 x^{2}+24 x y-12 x z-24 y z+18 z^{2}-12 x-24 y+36 z .
\end{aligned}
$$

Observe that $\mathcal{P}(s, t)$ satisfies the hypotheses of Theorem 2. Hence, a critical set for $\mathcal{P}(s, t)$ is the line $x=y=z$. On the other hand a representative of the inverse of $\mathcal{P}(s, t)$ is

$$
\begin{gathered}
\left\{s=-\frac{1}{12} \frac{x^{2}-4 x y+4 x z-4 y z+3 z^{2}-6 x+12 y-6 z-12}{y-z},\right. \\
\left.t=-\frac{1}{12} x^{2}+\frac{1}{3} y x-\frac{1}{3} z x+\frac{1}{3} z y-\frac{1}{4} z^{2}+\frac{1}{2} x-\frac{1}{2} z\right\} .
\end{gathered}
$$

Applying the method in Remark 2 we get as a critical set the variety

$$
V\left(y-z, x^{3}-x^{2} z-x z^{2}+z^{3}-6 x^{2}+12 x z-6 z^{2}-12 x+12 z\right)
$$

that decomposes as the line $y=x=z$ and the conic $V\left(y^{2}-6 y-x^{2}+6 x+12, y-z\right)$.

\section{Acknowledgements}

This work was developed, and partially supported, by the Spanish Ministerio de Economía y Competitividad under Project MTM2011-25816-C02-01. The first and third authors are members of the Research Group ASYNACS (Ref. CCEE2011/R34). 


\section{References}

1. C. Andradas, T. Recio, J. R. Sendra, and L. F. Tabera. On the simplification of the coefficients of a parametrization. J. Symbolic Comput., 44(2):192-210, 2009.

2. C. Andradas, T. Recio, J. R. Sendra, L. F. Tabera, and C. Villarino. Proper real reparametrization of rational ruled surfaces. Comput. Aided Geom. Design, 28(2):102-113, 2011.

3. Laurent Busé, David Cox, and Carlos D'Andrea. Implicitization of surfaces in $\mathbb{P}^{3}$ in the presence of base points. J. Algebra Appl., 2(2):189-214, 2003.

4. G. Bodnár, H. Hauser, J. Schicho, and O. Villamayor U. Plain varieties. Bull. Lond. Math. Soc., 40(6):965-971, 2008.

5. C. L. Bajaj and A. V. Royappa. Finite representations of real parametric curves and surfaces. Internat. J. Comput. Geom. Appl., 5(3):313-326, 1995.

6. F. Chen. Reparametrization of a rational ruled surface using the $\mu$-basis. Comput. Aided Geom. Design, 20(1):11-17, 2003.

7. D. Cox, J. Little, and D. O'Shea. Ideals, varieties, and algorithms. Undergraduate Texts in Mathematics. Springer, New York, third edition, 2007. An introduction to computational algebraic geometry and commutative algebra.

8. G. Farin, J. Hoschek, and M.-S. Kim, editors. Handbook of computer aided geometric design. North-Holland, Amsterdam, 2002.

9. J. Hoschek and D. Lasser. Fundamentals of computer aided geometric design. A K Peters Ltd., Wellesley, MA, 1993. Translated from the 1992 German edition by Larry L. Schumaker.

10. S. Pérez-Díaz and L.-Y. Shen. Characterization of rational ruled surfaces. J. Symbolic Comput., 2013. In press.

11. S. Pérez-Díaz, J. R. Sendra, and C. Villarino. A first approach towards normal parametrizations of algebraic surfaces. Internat. J. Algebra Comput., 20(8):977990, 2010 .

12. T. Recio, J. R. Sendra, L. F. Tabera, and C. Villarino. Generalizing circles over algebraic extensions. Math. Comp., 79(270):1067-1089, 2010.

13. J. Rafael Sendra. Normal parametrizations of algebraic plane curves. J. Symbolic Comput., 33(6):863-885, 2002.

14. T. Saito and T. W. Sederberg. Rational-ruled surfaces: implicitization and section curves. Graphical Models and Image Processing, 57(4):334-342, 1995.

15. J. R. Sendra, D. Sevilla, and C. Villarino. Covering of surfaces parametrized without projective base points. Submitted, 2014.

16. J. R. Sendra, D. Sevilla, and C. Villarino. Rational coverings of rational algebraic surfaces and removal of affine base points. Preprint, 2014. 